MATHEMATICS OF COMPUTATION

Volume 79, Number 270, April 2010, Pages 1091-1108

S 0025-5718(09)02283-2

Article electronically published on September 2, 2009

\title{
ON EQUATIONS OF DOUBLE PLANES WITH $p_{g}=q=1$
}

\author{
CARLOS RITO
}

\begin{abstract}
This paper describes how to compute equations of plane models of minimal Du Val double planes of general type with $p_{g}=q=1$ and $K^{2}=$ $2, \ldots, 8$. A double plane with $K^{2}=8$ having bicanonical map not composed with the associated involution is also constructed. The computations are done using the algebra system Magma.
\end{abstract}

\section{INTRODUCTION}

There are two typical methods to construct examples of surfaces: Campedelli - double covers ramified over curves, usually highly singular - and Godeaux quotients by group actions (cf. Rei91]). In this paper the first method is used to obtain new examples of double planes of general type with $p_{g}=q=1$. Often the singularities impose too many conditions on the linear parameters of systems of curves, and this implies hard calculations. The Computational Algebra System MAGMA ([BCP97]) is used to perform the computations.

Several authors have studied surfaces of general type with $p_{g}=q=1$ (Cat81, Cat99, CC91, CC93, CP06, Pol08, Pol09, Pol05, Pol06, Pig), but these surfaces are still not completely understood, and few examples are known.

For such a smooth minimal surface $S$, one has $2 \leq K^{2} \leq 9$ and $K^{2}=9$ only if the bicanonical map of $S$ (given by $|2 K|$ ) is birational (from CM02 and Xia85, Théorème 2.2]). Since $q=1$, the Albanese map of $S$ is a connected fibration onto an elliptic curve. We denote by $g$ the genus of a general Albanese fibre of $S$.

Du Val ([Du 52] ) classified the regular surfaces $S$ of general type with $p_{g} \geq 3$ whose general canonical curve is smooth and hyperelliptic. For these surfaces the bicanonical map is composed with an involution $i$ such that $S / i$ is rational. The families of surfaces exhibited by $\mathrm{Du}$ Val are nowadays called the Du Val examples.

The standard case of non-birationality of the bicanonical morphism $\phi_{2}$ is the case where $S$ has a genus 2 fibration.

For the non-standard case with $\phi_{2}$ of degree 2, bicanonical image a ruled surface and $p_{g}(S) \geq 1$, Gang Xiao ( Xia90, Theorem 2]) presented a list of possibilities which is a generalization of Du Val's list plus two extra families (this result is still valid assuming only that $\phi_{2}$ is composed with an involution such that the quotient surface is a ruled surface).

Received by the editor April 14, 2008 and, in revised form, March 26, 2009.

2000 Mathematics Subject Classification. Primary 14J29, 14Q05.

(C)2009 American Mathematical Society 
Later G. Borrelli ([Bor07]) excluded these two families and confirmed that, if the bicanonical map $\phi_{2}$ of $S$ is composed with $i, S / i$ is ruled and $S$ presents the non-standard case, then $S$ is a Du Val double plane (see Definition 3). But, to my knowledge, the existence of these double planes with $p_{g}=q=1$ and $K^{2}=4, \ldots, 7$ has not yet been shown, and no equation has been given for $K^{2}=3$ or 8 .

In this paper we compute equations of plane models of minimal Du Val double planes of general type with $p_{g}=q=1$ and $K^{2}=2, \ldots, 8$. We also construct a double plane with $K^{2}=8$ having bicanonical map not composed with the associated involution.

Notice that we give the first example of a minimal surface of general type with $p_{g}=q=1$ and $K^{2}=7$. We also give the first example with $K^{2}=5$ having Albanese fibration of genus $g \neq 2$.

The first example with $K^{2}=6$ has been given by the author in Rit07, as a double cover of a $K 3$ surface. Polizzi ( Pol09 $)$ also gives examples with $K^{2}=6,4$ and $g \neq 2$. These surfaces contain $8-K^{2}(-2)$-curves, all in the same Albanese fibre. One can verify that, in the double plane examples with $K^{2}=4$ or 6 , there is no Albanese fibre containing $8-K^{2}(-2)$-curves. Therefore our surfaces are different from the above examples.

In Pol06, Polizzi classifies surfaces of general type with $p_{g}=q=1, K^{2}=8$ and bicanonical map of degree 2 . He constructs examples using quotients under the action of a group and shows that these surfaces are Du Val double planes, describing the branch locus of the respective plane models. In sections 3.1 and 3.4 we show how to obtain equations for such branch loci.

Section 2 contains a structure description of the Albanese fibration of $S$ and the definition of a Du Val double plane. In Section 3 we describe the principal steps of the constructions. To obtain the equations of the branch loci we impose conditions to the linear parameters of systems of plane curves. Since the ramification curves are contained in Albanese fibres, in some cases it is easier to start by constructing non-reduced Albanese fibres, which simplify the computations. These are done using the algebra system Magma in Appendix A.

Notation and conventions. We work over the complex numbers; all varieties are assumed to be projective algebraic. For a projective smooth surface $S$, the canonical class is denoted by $K$, the geometric genus by $p_{g}:=h^{0}\left(S, \mathcal{O}_{S}(K)\right)$, the irregularity by $q:=h^{1}\left(S, \mathcal{O}_{S}(K)\right)$ and the Euler characteristic by $\chi=\chi\left(\mathcal{O}_{S}\right)=1+p_{g}-q$.

A $(-n)$-curve $C$ on a surface is a curve isomorphic to $\mathbb{P}^{1}$ such that $C^{2}=-n$. We say that a curve singularity is negligible if it is either a double point or a triple point which resolves to at most a double point after one blowup. An $\left(m_{1}, m_{2}, \ldots\right)$-point, or point of order $\left(m_{1}, m_{2}, \ldots\right)$, is a point of multiplicity $m_{1}$, which resolves to a point of multiplicity $m_{2}$ after one blowup, etc.

An involution of a surface $S$ is an automorphism of $S$ of order 2. We say that a map is composed with an involution $i$ of $S$ if it factors through the double cover $S \rightarrow S / i$.

The rest of the notation is standard in algebraic geometry.

\section{Preliminaries}

We say that a smooth surface $S$ is a double plane if $S$ has an involution $i$ such that $S / i$ is a rational surface. A plane model of $S$ is a double cover $X \rightarrow \mathbb{P}^{2}$ such 
that $X$ is a normal surface and there exists a commutative diagram

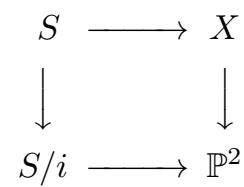

such that the horizontal arrows denote birational maps.

2.1. Double planes with $q=1$. Let $S$ be a smooth minimal surface of general type with an involution $i$. Since $S$ is minimal of general type, this involution is biregular. The fixed locus of $i$ is the union of a smooth curve $R^{\prime \prime}$ (possibly empty) and of $t \geq 0$ isolated points $P_{1}, \ldots, P_{t}$. Let $S / i$ be the quotient of $S$ by $i$ and $p: S \rightarrow S / i$ be the projection onto the quotient. The surface $S / i$ has nodes at the points $Q_{i}:=p\left(P_{i}\right), i=1, \ldots, t$, and is smooth elsewhere. If $R^{\prime \prime} \neq \emptyset$, the image via $p$ of $R^{\prime \prime}$ is a smooth curve $B^{\prime \prime}$ not containing the singular points $Q_{i}, i=1, \ldots, t$. Now let $h: V \rightarrow S$ be the blowup of $S$ at $P_{1}, \ldots, P_{t}$ and set $R^{\prime}=h^{*}\left(R^{\prime \prime}\right)$. The involution $i$ induces a biregular involution $\widetilde{i}$ on $V$, whose fixed locus is $R:=R^{\prime}+\sum_{1}^{t} h^{-1}\left(P_{i}\right)$, and the quotient $W:=V \widetilde{i}$ is smooth.

Let $B$ be the branch locus of the projection $\pi: V \rightarrow W$, i.e. the image of $R$ on $W$. Let $L$ be the line bundle such that $2 L \equiv B$ and $B, L$ determine $\pi$. We have

$$
H^{0}\left(V, \mathcal{O}_{V}\left(2 K_{V}\right)\right) \cong H^{0}\left(W, \mathcal{O}_{W}\left(2 K_{W}+B\right)\right) \oplus H^{0}\left(W, \mathcal{O}_{W}\left(2 K_{W}+L\right)\right)
$$

The first and second summands correspond, respectively, to the invariant and antiinvariant parts of the bicanonical system of $V$.

Suppose now that $q(S)=1$ and that $S / i$ is a rational surface. Since $q=1$, the Albanese variety of $S$ is an elliptic curve $E$ and the Albanese map is a connected fibration (see e.g. [Bea78] or [BPV84]). This fibration is preserved by $i$ and so we have a commutative diagram

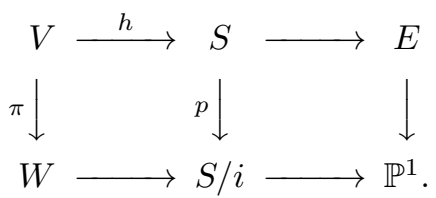

Denote by

$$
f_{A}: W \rightarrow \mathbb{P}^{1}
$$

the fibration induced by the Albanese fibration of $S$. The double cover $E \rightarrow \mathbb{P}^{1}$ is ramified over 4 points $p_{j}$ of $\mathbb{P}^{1}$; thus the branch locus $B$ of $\pi$ is contained in 4 fibres

$$
F_{A}^{j}:=f_{A}^{*}\left(p_{j}\right), j=1, \ldots, 4,
$$

of the fibration $f_{A}$. By Zariski's Lemma (see e.g. [BPV84]) the irreducible components $B_{i}$ of $B$ satisfy $B_{i}^{2} \leq 0$. Since $\pi^{*}\left(F_{A}^{j}\right)$ has even multiplicity, each component of $F_{A}^{j}$ which is not a component of the branch locus must be of even multiplicity.

In the following sections $W$ can always be contracted to $\mathbb{P}^{2}$. We keep the same notation for the image of $f_{A}$ and $F_{A}^{j}$ on $\mathbb{P}^{2}$. 


\subsection{Du Val double planes.}

Notation 1 . Given distinct points $p_{0}, \ldots, p_{j}, \ldots, p_{j+s} \in \mathbb{P}^{2}$, let $T_{i}$ denote the line through $p_{0}$ and $p_{i}, i=1, \ldots, j$. We say that a plane curve is of type

$$
d\left(m,(n, n)_{T}^{j}, r^{s}\right)
$$

if it is of degree $d$ and if it has an $m$-tuple point at $p_{0}$, an $(n, n)$-point at $p_{1}, \ldots, p_{j}$, an $r$-tuple point at $p_{j+1}, \ldots, p_{j+s}$ and no other non-negligible singularities. The index $_{T}$ is used if $T_{i}$ is tangent to the $(n, n)$-point at $p_{i}$.

An obvious generalization is used if there are other singularities.

Definition 2. Let $C_{0}$ and $F$ denote, respectively, the negative section and a rational fibre of the Hirzebruch surface $\mathbb{F}_{2}$.

The Bombieri-Du Val surface is the minimal model of the double cover of $\mathbb{F}_{2}$ with branch locus a smooth curve in $C_{0}+\left|7 C_{0}+14 F\right|$.

Definition 3. A Du Val surface is either

$\mathcal{B})$ the Bombieri-Du Val surface

or a double plane having a plane model $X \rightarrow \mathbb{P}^{2}$ with branch locus $D$ one of the following:

$\mathcal{D})$ a smooth curve of degree 8 ;

$\left.\mathcal{D}_{0}\right)$ a smooth curve of degree $10 ;$

$\left.\mathcal{D}_{n}\right)$ a curve of type $[10+2 n]\left(2 n+2,(5,5)_{T}^{n}\right), n \in\{1, \ldots, 6\}$;

or one of $\mathcal{B}, \mathcal{D}$ or $\mathcal{D}_{n}$, imposing additional singularities to $D$ which correspond, on the branch locus of the canonical resolution of $X$, to negligible singularities, 4-tuple points or $(3,3)$-points.

Surfaces of type $\mathcal{B}, \mathcal{D}$ or $\mathcal{D}_{n}$ are called Du Val's ancestors. Du Val surfaces are also called $D u$ Val double planes. We have the following:

Theorem 4 ([Bor07]). Let $S$ be a smooth minimal surface of general type. Suppose that $S$ has no pencil of genus-2 curves. Then the following conditions are equivalent:

a) the bicanonical map of $S$ factors through a rational map of degree two $\phi$ : $S \rightarrow \Sigma$, where $\Sigma$ is a rational or ruled surface;

b) $S$ is the smooth minimal model of a Du Val double plane.

Moreover, if $S$ is as in $\mathrm{b})$, then $q(S)=0$ unless $p_{g}(S)=q(S)=1$.

Notice that the imposition of a 4-tuple point to the branch locus decreases $K^{2}$ by 2 and the Euler characteristic $\chi$ by 1 , while a $(3,3)$-point decreases both $K^{2}$ and $\chi$ by 1 . Negligible singularities in the branch locus do not change these invariants.

The imposition of $6-n$ 4-tuple or $(3,3)$-points to the branch locus of a Du Val surface of type $\mathcal{D}_{n}$ gives $p_{g}=q=1$ if there is exactly one conic through the singular points $p_{1}, \ldots, p_{6}$.

In the case $\mathcal{D}_{n}$, the branch locus contains the lines $T_{1}, \ldots, T_{n}$. Each of these lines corresponds to two $(-2)$-curves in $W$, which contract to two nodes of $S / i$. A $(3,3)$-point in the branch locus also corresponds to a node of $S / i$.

The bicanonical map of a Du Val double plane of general type factors through a map of degree 2 onto a rational surface because the anti-invariant part of the bicanonical system is empty. Moreover, using (1) of Section 2.1 and using that $h^{0}\left(S, \mathcal{O}_{S}\left(2 K_{S}\right)\right)=K_{S}^{2}+\chi(S)$ for $S$ minimal of general type (see e.g. BPV84, Ch. VII, Cor. 5.4]), one can show the following: 
Proposition 5. Let $S$ be the minimal model of a Du Val double plane obtained by imposing $(3,3)$ or 4-tuple points to the branch locus $D$ of a Du Val's ancestor of type $\mathcal{D}_{n}$. Let $a, b$ be the number of such points, respectively.

If $S$ is a surface of general type, then $K_{S}^{2}+\chi(S)$ is equal to the number of generators of the linear system

$$
\left|(4+2 n) \psi^{*}(H)-2 n E_{0}-\sum_{1}^{n}\left(2 E_{i}+4 E_{i}^{\prime}\right)-\sum_{n+1}^{n+a} 2 E_{i}^{\prime}-\sum_{n+a+1}^{n+a+b} 2 E_{i}\right|,
$$

where $\psi$ is the blowup of $\mathbb{P}^{2}$ which resolves the singularities of $D, H$ is a line in $\mathbb{P}^{2}$, $E_{0}, \ldots, E_{n+a+b}$ are exceptional divisors with self-intersection -1 and $E_{1}^{\prime}, \ldots, E_{n+a}^{\prime}$ are (-1)-curves corresponding to the blowup at the infinitely near points.

For more information on Du Val surfaces, see [Du 52, Cil97] or [Bor07.

2.3. The Magma procedures LinSys and LinSys2. Magma has the function LinearSystem, which calculates linear systems of plane curves with ordinary singularities, but we want to work with non-ordinary singularities. To achieve this, we define two procedures. The first one, LinSys, calculates the linear system $L$ of plane curves of degree $d$, in an affine plane $\mathbb{A}$, having singular points $p_{i}$ of order $\left(m 1_{i}, m 2_{i}\right)$ with tangent slope $t d_{i}$.

The other procedure, LinSys2, calculates the subsystem $J$, of a given linear system $L$ of plane curves, of those sections which have a singularity, at a point $q$, of type $m=\left(m_{1}, \ldots, m_{j}\right)$ with tangent slopes given by $t d=\left[t d_{1}, \ldots, t d_{j-1}\right]$.

The code lines for these procedures are in Appendix A.1

\section{Equations of Plane models With $p_{g}=q=1$}

In this section we obtain equations of the branch locus $B \subset \mathbb{P}^{2}=\mathbb{P}(1,1,1)$ of minimal Du Val double planes $S$, with ancestor of type $\mathcal{D}_{n}$ (see Definition 3), with $p_{g}=q=1$ and $K^{2}=2, \ldots, 8$. Notice that if $f=0$ is the defining equation of $B$, then the equation $w^{2}=f$ gives a plane model of $S$ in the weighted projective space $\mathbb{P}((\operatorname{deg} f) / 2,1,1,1)$.

In Section 3.5 we construct a double plane with $K^{2}=8$ having bicanonical map not composed with the associated involution.

In order to obtain $q=1$, all the singularities, except the one at $p_{0}$, are chosen to be contained in exactly one conic.

Let $W, L$ be as in Section 2.1. In each case we compute

$$
K^{2}=2\left(K_{W}+L\right)^{2}+t,
$$

where $t$ is the number of $(-1)$-curves contained in the ramification divisor. We get $K^{2}>0$. Since also $p_{g}>0, S$ is of general type. To exclude the possibility of hidden (-1)-curves, we use the Magma procedure LinSys defined in Appendix A.1 to compute the dimension of the linear system given in Proposition 5. This returns the same value for $K^{2}$, which implies the minimality of the surface.

Table 1 lists the type of each branch curve that we are going to obtain and the corresponding values of $\left(K^{2}, g\right)$, where $g$ denotes the genus of a general Albanese fibre of $S$.

We keep Notation 1 .

First we find double planes with $\left(K^{2}, g\right)=(8,5)$ and $\left(K^{2}, g\right)=(7,5)$. In the first case the branch locus contains an element of the pencil $f_{A}$ which induces the 
TABLE 1

\begin{tabular}{c|c} 
Type of branch curve & $\left(K^{2}, g\right)$ \\
\hline $22\left(14,(5,5)_{T}^{6}\right)$ & $(8,5),(8,4),(8,3)$ \\
{$[10+2 n]\left(2 n+2,(5,5)_{T}^{n}, 4^{6-n}\right)$} & $(6,4),(4,3),(2,2)$ \\
$n=5,4,3$ & \\
{$[10+2 n]\left(2 n+2,(5,5)_{T}^{n},(3,3), 4^{5-n}\right)$} & $(7,5),(5,4),(3,3)$ \\
$n=5,4,3$ & $(6,3)$ \\
$18\left(10,(5,5)_{T}^{4},(3,3)^{2}\right)$ & $(8,4)$ \\
$26\left(14,(7,7)_{T}^{5}\right)$ &
\end{tabular}

Albanese fibration. From here constructions with $\left(K^{2}, g\right)=(6,4),(5,4),(4,3)$, $(3,3),(2,2)$ will follow easily. A construction with $\left(K^{2}, g\right)=(6,3)$ and branch locus strictly contained in elements of $f_{A}$ is also given. Then we get surfaces with $\left(K^{2}, g\right)=(8,4)$ or $(8,3)$. These three surfaces with $K^{2}=8$ are surfaces of type I, II, III described by F. Polizzi in Pol06. Finally we construct a pencil $l$ of plane curves and a double plane with branch locus containing an element of $l$ having $K^{2}=8$ and bicanonical map not composed with the corresponding involution.

A difficulty that arises in the computation of singular curves is that often the computer is not able to finish the calculations. Except for Section 3.2, our method is to try to figure out the configuration of the non-reduced fibres $F_{A}^{i}$ of $f_{A}$, described in Section 2.1. The problem of finding the support of $F_{A}^{i}$ deals with curves of lower degree and with simpler singularities, hence with faster computations.

The next sections of this chapter describe the principal steps. The detailed calculations are done in Appendix A, using the Computational Algebra System Magma.

We keep Notation 1 .

3.1. $K^{2}=8,6,4,2$ and $g=5,4,3,2$. To construct a Du Val's ancestor $S$ of type $\mathcal{D}_{6}$ (it has $K^{2}=8$ ) it suffices to find a curve $B^{\prime}$ of type

$$
16\left(8,(4,4)_{T}^{6}\right) \text {, }
$$

singular at $p_{0}, \ldots, p_{6}$, such that the branch locus $B:=B^{\prime}+\sum_{1}^{6} T_{i}$ is reduced. If $p_{1}, \ldots, p_{6}$ are contained in exactly one conic, then $p_{g}(S)=q(S)=1$.

In this example the pencil $f_{A}$ which induces the Albanese fibration of $S$ has the following elements:

$$
F_{A}^{1}=2 D_{1}+T_{3}+\cdots+T_{6}, \quad F_{A}^{2}=2 D_{2}+T_{1}+T_{2}, \quad F_{A}^{3}=2 D_{3}, \quad F_{A}^{4}=D_{4},
$$

where $D_{1}, \ldots, D_{4}$ are curves of types

$$
6\left(2,(2,2)_{T}^{2},(2,1)_{T}^{4}\right), \quad 7\left(3,(2,1)_{T}^{2},(2,2)_{T}^{4}\right), \quad 8\left(4,(2,2)_{T}^{6}\right), \quad 16\left(8,(4,4)_{T}^{6}\right),
$$

respectively.

To construct $B^{\prime}$, we first find points $p_{0}, \ldots, p_{6}$ such that $D_{1}$ exists. Then we use the procedure LinSys (see Section 2.3) to verify the existence of $D_{2}$ and $D_{3}$, also singular at $p_{0}, \ldots, p_{6}$. The curve $B^{\prime}=D_{4}$ is a general element of the pencil generated by $F_{A}^{1}, F_{A}^{2}, F_{A}^{3}$. 
So let us look for $D_{1}$. Briefly, the steps are as follows. Let $\mathbb{A}$ be an affine plane, $C$ be a smooth conic not containing the origin $p_{0}$ of $\mathbb{A}$ and $p_{1}, \ldots, p_{4}$ be points in $C$. Denote by $L$ the linear system of plane curves of type

$$
6\left(2,(2,2)_{T}^{2},(2,1)_{T}^{2}\right)
$$

with singularities at $p_{0}, \ldots, p_{4}$, respectively. Let $F$ be a general element of $L$ and $p_{5}, p_{6}$ be general points of $\mathbb{A}$. We define a scheme $S c h$ by imposing the following conditions:

- $p_{5}, p_{6} \in C \cap F$;

- $p_{5}, p_{6}$ are double points of $F$;

- the singularities of $F$ at $p_{5}, p_{6}$ have one branch tangent to $T_{5}, T_{6}$;

- $p_{5} \neq p_{6}$ and $p_{5}, p_{6} \notin\left\{p_{0}, \ldots, p_{4}\right\}$.

Now we compute the points of $S c h$ with Magma, choosing one of the solutions for $p_{5}, p_{6}$, and we use the procedure LinSys to compute $B^{\prime}$, a general element of the pencil of curves of type $16\left(8,(4,4)_{T}^{6}\right)$, with singularities at $p_{0}, \ldots, p_{6}$.

Finally we perform some verifications: that $B^{\prime}$ is reduced, the singularities are as expected, the value of $K_{S}^{2}$ is as claimed, etc.

With this we find a minimal double plane with $p_{g}=q=1$ and $K^{2}=8$. The divisor $2 D_{1}+T_{3}+\cdots+T_{i}$ is also a good candidate for one of the singular fibres $F_{A}^{i}$ in the case where the branch locus is a curve of type

$$
[10+2 n]\left(2 n+2,(5,5)_{T}^{n}, 4^{6-n}\right), \quad n=5,4,3 .
$$

In fact, using the points $p_{0}, \ldots, p_{6}$ and the procedure LinSys, one can find branch loci of those types, obtaining then minimal double planes with $p_{g}=q=1$, $K^{2}=6,4,2$ and $g=4,3,2$, respectively.

The corresponding Magma calculations are in Appendix A.2.1 There we use symmetry in order to obtain faster computations.

3.2. $K^{2}=7,5,3$ and $g=5,4,3$. Here we impose a $(3,3)$-point to the branch locus of a Du Val's ancestor of type $\mathcal{D}_{5}$; i.e., we construct a plane curve $B^{\prime}$ of type

$$
15\left(7,(4,4)_{T}^{5},(3,3)\right)
$$

such that $B:=B^{\prime}+\sum_{1}^{5} T_{i}$ is reduced (see Notation 1). The double cover with branch locus $B$ is a plane model of a Du Val double plane $S$ with $K^{2}=7$ and $\chi=1$.

In this example the $(3,3)$-point $p_{6}$ is infinitely near to the $(5,5)$-point $p_{1}$ of $B$ (i.e., there is a $(5,5,3,3)$-point at $\left.p_{1}\right)$ and the line $T_{1}$, through $p_{0}, p_{1}$, is tangent to the conic $C$ defined by $p_{1}, \ldots, p_{5}$. This implies that $q(S)=1$, because $\widetilde{C}-\sum_{1}^{6} E_{i}$ is effective, where the curves $E_{i}$ are the exceptional divisors corresponding to the blowups at the points $p_{i}$, and $\widetilde{C}$ is the pullback of $C$.

In this case the pencil $f_{A}$ has elements

$$
F_{A}^{1}=2 D_{1}+T_{4}+T_{5}, \quad F_{A}^{2}=2 D_{2}+T_{2}+T_{3}, \quad F_{A}^{3}=2 D_{3}, \quad F_{A}^{4}=D_{4}+T_{1},
$$


where $D_{1}, \ldots, D_{4}$ are curves of types

$$
\begin{aligned}
& 7\left(3,(2,2)_{T}^{3},(2,1)_{T}^{2},(2,2)\right), \quad 7\left(3,(2,2)_{T},(2,1)_{T}^{2},(2,2)_{T}^{2},(2,2)\right), \\
& 8\left(4,(2,2)_{T}^{5},(2,2)\right), \quad 15\left(7,(4,4)_{T}^{5},(3,3)\right),
\end{aligned}
$$

respectively.

To find $B^{\prime}=D_{4}$ we proceed as follows. In an affine plane $\mathbb{A}$, we fix a smooth conic $C$ not containing the origin $p_{0}$ and we choose distinct points $p_{1}, \ldots, p_{5} \in C$ such that $T_{1}$ is tangent to $C$ at $p_{1}$. We compute the linear system $L$ of plane curves of type $15\left(7,(4,4)_{T}^{5}\right)$ and we resolve the base point of $L$ at $p_{1}$, denoting the resulting linear system by $L_{1}$. The defining polynomial of $B^{\prime}$ is given by (the blowdown of) a linear combination of elements of $L_{1}$.

In order to obtain a $(3,3)$-point $p_{6}$ (infinitely near to $p_{1}$ ), we impose conditions, given by the zeros of the derivatives up to order 2 , to the elements of $L_{1}$. Then we resolve this point and impose another triple (infinitely near) point. With all these conditions we define a matrix, which is denoted by $M t$ in Appendix A.2.2. To have a solution it is necessary that $M t$ has no maximal rank.

We define a scheme $S$ ch by imposing the following conditions:

- the vanishing of the maximal minors of $M t$;

- the points are infinitely near.

Now we compute the points of $S c h$ with Magma, choosing one of the solutions for $p_{6}$, and we use the procedures of Section 2.3 to compute $B^{\prime}$, with singularities at $p_{0}, \ldots, p_{6}$.

Finally we perform some verifications. In particular we show that $B^{\prime}$ is reduced, the singularities are as expected, there are no other singularities and the value of $K_{S}^{2}$ is as claimed.

To find the pencil which induces the Albanese fibration, one uses again the procedures LinSys and LinSys2 to compute the pencil of curves of type

$$
16\left(8,(4,4)_{T}^{5},(4,4)\right) \text {, }
$$

through $p_{0}, \ldots, p_{6}$, and to verify that the elements $F_{A}^{1}, \ldots, F_{A}^{4}$ are as claimed.

With this we find a minimal double plane with $p_{g}=q=1, K^{2}=7$ and $g=5$. Using the above Magma procedures, one can verify that there exist also branch loci of type

$$
18\left(10,(5,5)_{T}^{4}, 4,(3,3)\right) \text { and } 16\left(8,(5,5)_{T}^{3}, 4^{2},(3,3)\right),
$$

through $p_{0}, \ldots, p_{6}$. These correspond to minimal Du Val double planes with $p_{g}=$ $q=1$ and $\left(K^{2}, g\right)=(5,4),(3,3)$. The pencils which induce the Albanese fibration are of type

$$
15\left(7,(4,4)_{T}^{4}, 4,(4,4)\right) \text { and } 14\left(6,(4,4)_{T}^{3}, 4^{2},(4,4)\right),
$$

respectively.

The Magma calculations for this section are in Appendix A.2.2.

3.3. $K^{2}=6$ and $g=3$. In this section we impose two (3,3)-points to the branch locus of a Du Val's ancestor of type $\mathcal{D}_{4}$; i.e., we construct a plane curve $B^{\prime}$ of type

$$
14\left(6,(4,4)_{T}^{4},(3,3)^{2}\right),
$$


singular at points $p_{0}, \ldots, p_{6}$, such that $B:=B^{\prime}+\sum_{1}^{4} T_{i}$ is reduced. In this example the $(3,3)$-points $p_{5}, p_{6}$ are tangent to the conic $C$ through $p_{1}, \ldots, p_{6}$. The double cover with branch locus $B$ is a plane model of a Du Val double plane $S$ with $K^{2}=6$, $p_{g}=q=1$ and $g=3$.

The pencil $f_{A}$ which induces the Albanese fibration of $S$ contains four fibres

$$
F_{A}^{1}=D_{1}+2 T_{0}, \quad F_{A}^{2}=D_{2}, \quad F_{A}^{3}=2 D_{3}, \quad F_{A}^{4}=2 C+T_{1}+\cdots+T_{4},
$$

where $T_{0}$ is a line through $p_{0}, p_{5}, p_{6}$, and $D_{1}, D_{2}, D_{3}$ are curves of types

$$
6\left(2,(2,2)_{T}^{4},(1,1)^{2}\right), \quad 8\left(4,(2,2)_{T}^{4},(2,2)^{2}\right), \quad 4\left(2,(1,1)_{T}^{4},(1,1)^{2}\right),
$$

respectively. We have

$$
B^{\prime}=D_{1}+D_{2} .
$$

First we construct $D_{1}$ and then we use the procedure LinSys of Section 2.3 to obtain $D_{2}$ (a general element of $f_{A}$ ) and to verify the existence of $D_{3}$.

To find $D_{1}$, we follow the steps used in Section 3.1. but now with $L$ the linear system of curves of type $6\left(2,(2,2)_{T}^{4}\right)$ and $S c h$ defined by

- $p_{5}, p_{6} \in C \cap F$

- $F$ is smooth at $p_{5}, p_{6}$;

- $F$ is tangent to $C$ at $p_{5}, p_{6}$;

- $p_{5} \neq p_{6}$ and $p_{5}, p_{6} \notin\left\{p_{0}, \ldots, p_{4}\right\}$.

The details can be found in Appendix A.2.3. Again we use symmetry in order to increase the speed of the calculations.

3.4. $K^{2}=8$ and $g=4$ or 3 . Given, in an affine plane $\mathbb{A}$, a general plane cubic $Q$ not containing the origin $p_{0}$, there are 6 points $p_{1}, \ldots, p_{6} \in Q$ such that each line $T_{i}$, defined by $p_{0}, p_{i}$, is tangent to $Q$. In this example we have

$$
F_{A}^{1}=3 D_{1}, \quad F_{A}^{2}=D_{2}, \quad F_{A}^{3}=2 D_{3}, \quad F_{A}^{4}=2 Q+T_{1}+\cdots+T_{6},
$$

where $D_{1}, D_{2}, D_{3}$ are curves of types

$$
4\left(2,(1,1)_{T}^{6}\right), \quad 12\left(6,(3,3)_{T}^{6}\right), \quad 6\left(3,(2,1)_{T}^{6}\right),
$$

respectively.

Using the procedure LinSys (see Section 2.3), one can verify the existence of the curves $D_{1}, D_{2}, D_{3}$, through $p_{0}, \ldots, p_{6}$, and that $p_{1}, \ldots, p_{6}$ are contained in exactly one conic. The double cover with branch locus $D_{1}+D_{2}+\sum_{1}^{6} T_{i}$ is a plane model of a Du Val double plane $S$ with $K^{2}=6, p_{g}=q=1$ and $g=4$. This is a surface of type II described in Pol06.

Finally the surface of type I described in Pol06:

Let $C \subset \mathbb{P}^{2}$ be a smooth conic and $p_{0} \notin C, p_{1}, \ldots, p_{6} \in C$ be points such that the lines $T_{5}$ and $T_{6}$, through $p_{0}, p_{5}$ and $p_{0}, p_{6}$, are tangent to $C$. One can verify the existance of a smooth cubic $D$ of type $3\left(1,(1,1)_{T}^{4}, 1^{2}\right)$, through $p_{0}, \ldots, p_{6}$. Let

$$
F_{A}^{1}=2 C+T_{1}+\cdots+T_{4}, \quad F_{A}^{2}=2 D+T_{5}+T_{6}
$$

and $F_{A}^{3}, F_{A}^{4}$ be general elements of the pencil generated by $F_{A}^{1}$ and $F_{A}^{2}$. The branch locus in this case is

$$
F_{A}^{3}+F_{A}^{4}+\sum_{1}^{6} T_{i}
$$


3.5. $K^{2}=8, g=4, \phi_{2}$ not composed with $i$. This section describes the steps taken to obtain an equation of a double plane $S$ of general type with $K^{2}=8$, $p_{g}=q=1$ and Albanese fibres of genus $g=4$, such that the bicanonical map $\phi_{2}$ of $S$ is not composed with the associated involution $i$ of $S$.

In this example the pencil $f_{A}$ which induces the Albanese fibration has four fibres

$$
F_{A}^{1}=2 D_{1}+T_{5}, \quad F_{A}^{2}=6 D_{2}+3 T_{1}, \quad F_{A}^{3}=2 D_{3}+T_{2}+T_{3}+T_{4}, \quad F_{A}^{4}=D_{4},
$$

where $D_{1}, \ldots, D_{4}$ are curves of types

$$
\begin{array}{lr}
10\left(4,(3,3)_{T}^{4},(3,2)_{T}\right), & 3\left(1,1,(1,1)_{T}^{4}\right), \\
9\left(3,(3,3),(3,2)_{T}^{3},(3,3)\right), & 21\left(9,(6,6)_{T}^{5}\right),
\end{array}
$$

respectively. The surface $S$ is the minimal double plane given by the branch locus

$$
D_{4}+\sum_{1}^{5} T_{i}
$$

From the double cover formulas ([BPV84, Section V. 22]), one obtains $K_{S}^{2}=8$ (notice that the pullback of the lines $T_{i}$ contains $10(-1)$-curves), $\chi\left(\mathcal{O}_{S}\right)=1$ and $p_{g}(S)$ is equal to the number of generators of the linear system of curves of type $10\left(6,(2,3)_{T}^{5}\right)$.

The points $p_{0}, \ldots, p_{5}$ are chosen such that the line $T_{1}$, through $p_{0}, p_{1}$, is tangent to the conic defined by $p_{1}, \ldots, p_{5}$. One can verify that this implies the existence of the cubic $D_{2}$. After finding $D_{1}$, we verify the existence of $D_{2}$ and $D_{3}$. The curve $D_{4}$ is a general element of the pencil generated by $F_{A}^{1}, F_{A}^{2}, F_{A}^{3}$.

The difficulty here is the computation of $D_{1}$. This is done following the steps of Section 3.2. We omit the details.

We notice that the procedure LinSys can be used to compute the number of elements of the linear system of curves of type $10\left(6,(2,3)_{T}^{5}\right)$, showing that $p_{g}(S)=1$, and to compute the invariant and anti-invariant parts of the bicanonical system (see Section 2.1), showing the above claim about the bicanonical map of $S$ and confirming that $K_{S}^{2}=8$.

\section{Appendix A. Magma computations}

In this appendix several computations are done using the Computational Algebra System MAGMA.

The following functions will be useful:

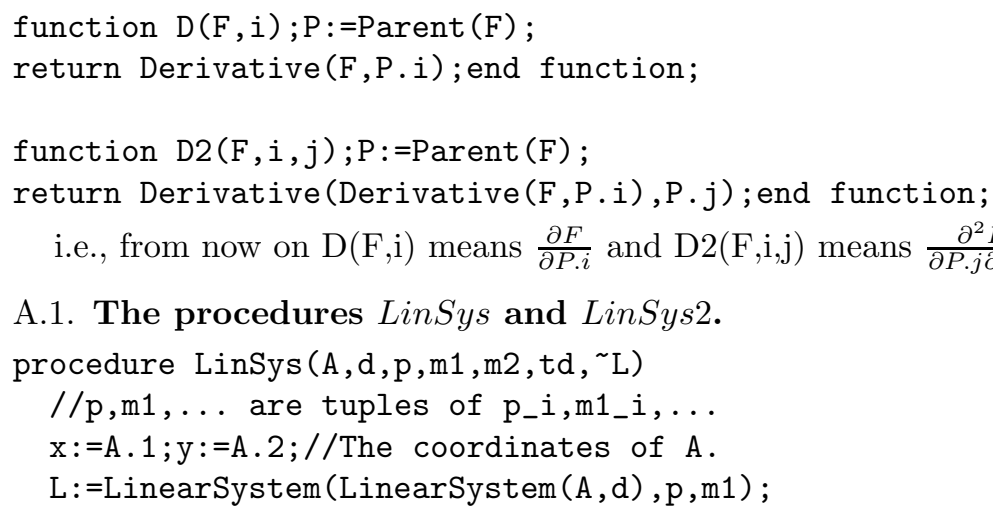

A.1. The procedures LinSys and LinSys2. 


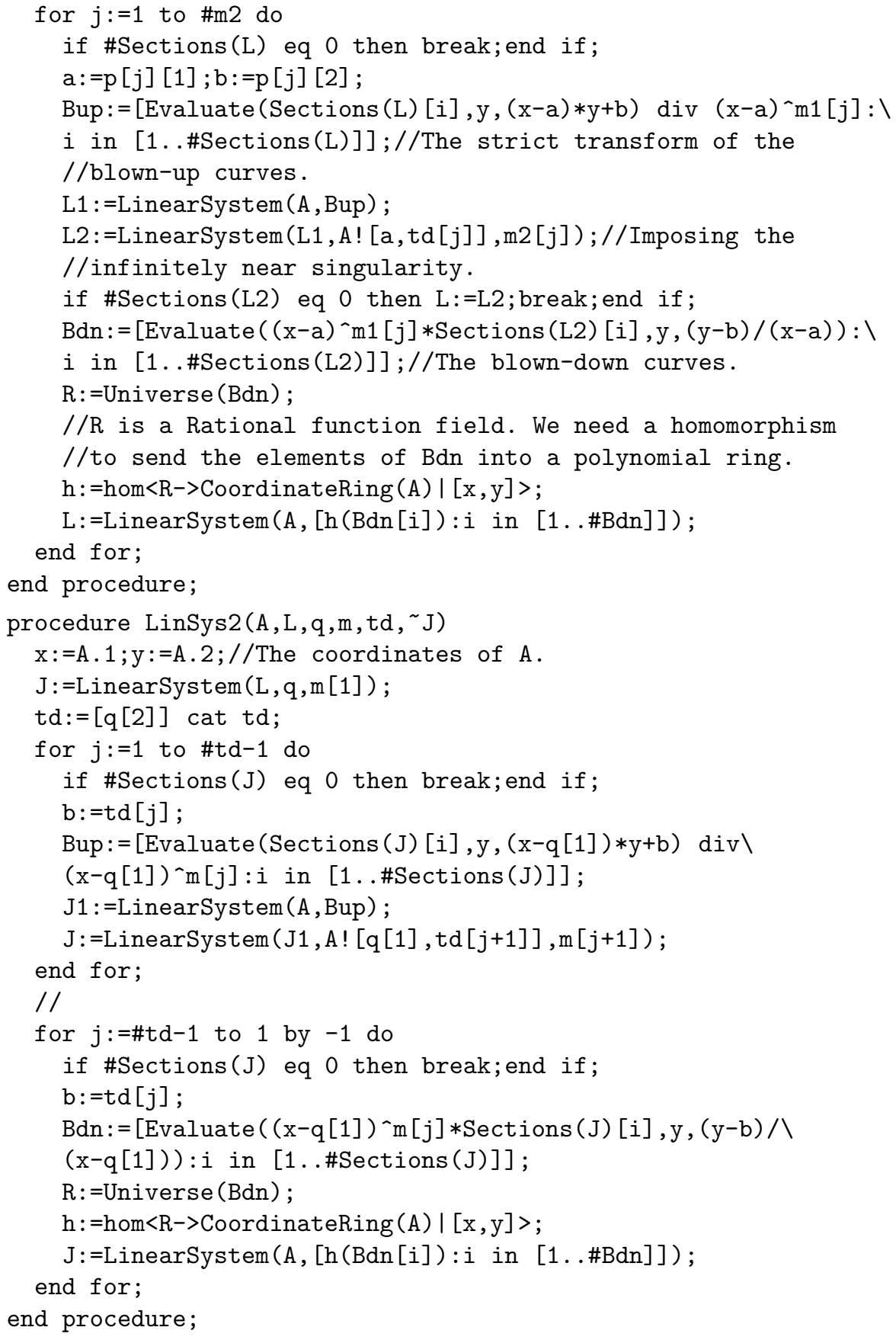

\section{A.2. Double planes.}

A.2.1. $K^{2}=8,6,4,2$ and $g=5,4,3,2$. Here we have the computations of Section 3.1. with details for the case of a double plane with $p_{g}=q=1$ and $K^{2}=6$.

$>\mathrm{A}\langle\mathrm{x}, \mathrm{y}\rangle:=$ AffineSpace (Rationals ()$, 2)$;

$>\mathrm{p}:=[\mathrm{A} ![7 / 5,4 / 5], \mathrm{A} ![7 / 5,-4 / 5], \mathrm{A} ![2,1], \mathrm{A} ![2,-1], \operatorname{Origin}(\mathrm{A})]$; 


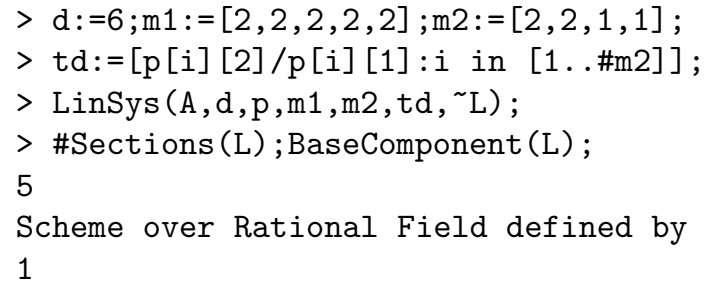

The condition $e q F=0$ is used to obtain one branch of the double point $p_{5}=(x, y)$ tangent to the line $T_{5}$. In fact, the tangent cone of a plane curve $\{F(X, Y)=0\}$ at $(x, y)$ is given by

$$
\frac{\partial^{2} F}{\partial X^{2}}(x, y) \frac{(X-x)^{2}}{2}+\frac{\partial^{2} F}{\partial X \partial Y}(x, y)(X-x)(Y-y)+\frac{\partial^{2} F}{\partial Y^{2}}(x, y) \frac{(Y-y)^{2}}{2} .
$$

One can verify that this equation is divisible by $x Y-y X$ only if

$$
\begin{gathered}
2 x y \frac{\partial^{2} F}{\partial X \partial Y}(x, y)+x^{2} \frac{\partial^{2} F}{\partial X^{2}}(x, y)+y^{2} \frac{\partial^{2} F}{\partial Y^{2}}(x, y)=0 . \\
>\operatorname{dif}:=\mathrm{y} *(\mathrm{y}-1 / 2 * \mathrm{x}) *(\mathrm{y}-4 / 7 * \mathrm{x}) *(\mathrm{y}+1 / 2 * \mathrm{x}) *(\mathrm{y}+4 / 7 * \mathrm{x}) ;
\end{gathered}
$$

In order to obtain $p_{5}, p_{6} \notin T_{i}, i=1, \ldots, 4$, and $p_{5} \neq p_{6}$, we need dif to be different from zero. This is achieved by imposing the condition $1+n \cdot d i f=0$.

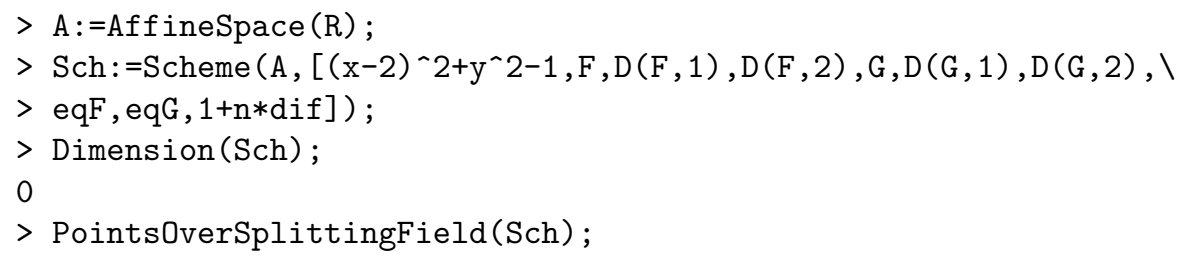

This command gives the points of $S c h$ and the necessary field extensions to define them. Choosing one of the solutions we obtain points $p_{5}, p_{6}$ such that there exists a pencil $f_{A}$ of curves of type $15\left(7,(4,4)_{T}^{5}, 4\right)$. Let $B^{\prime}$ be a general element of $f_{A}$. The branch locus $B:=B^{\prime}+\sum_{1}^{5} T_{i}$ is of type $20\left(12,(5,5)_{T}^{5}, 4\right)$. The corresponding minimal double plane is a surface of general type with $p_{g}=q=1, K^{2}=6$ and $g=4$.

Verification that $B_{0}:=B^{\prime}$ is as stated:

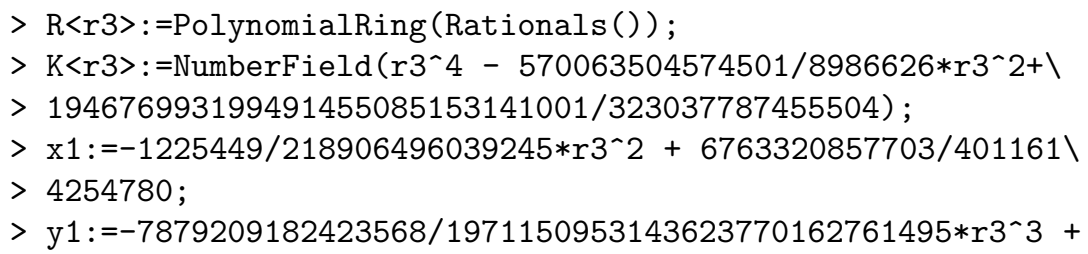




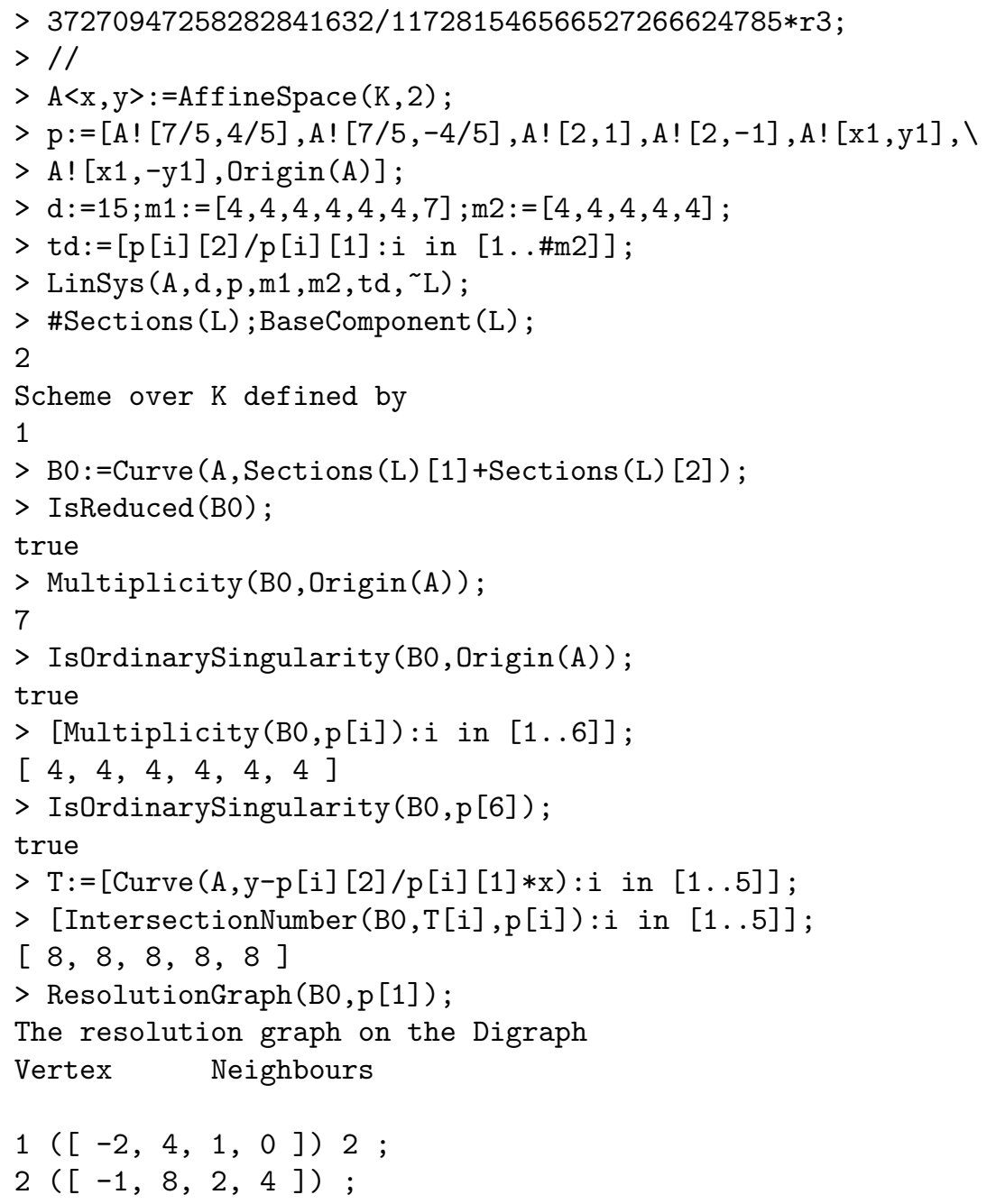

One obtains the same resolution graph for $p_{2}, \ldots, p_{5}$.

The curve $B_{0}$ has been chosen as a general element of $L$. Since this linear system has no base component and no additional base points (notice that the resolution of an element of $L$ at the above base points has self-intersection zero), $B_{0}$ has no other singularities.

Now we show that the number of generators of the linear system described in Proposition 5 is 7 , confirming that $K^{2}=6$.

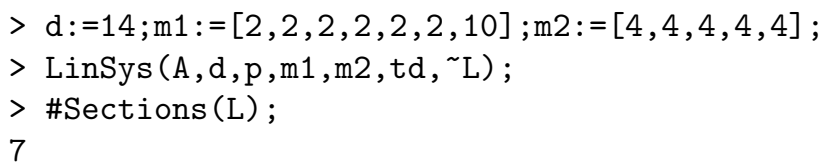

The other cases, $p_{g}=q=1, K^{2}=8,4,2$ and $g=5,3,2$, are analogous to the previous one. One needs only to ask Magma (using the procedure LinSys) for 
curves of type

$$
[10+2 n]\left(2 n+2,(5,5)_{T}^{n}, 4^{6-n}\right), \quad n=6,4,3,
$$

with singularities at the previous points $p_{0}, \ldots, p_{6}$.

A.2.2. $K^{2}=7,5,3$ and $g=5,4,3$. The detailed computations of Section 3.2 are as follows.

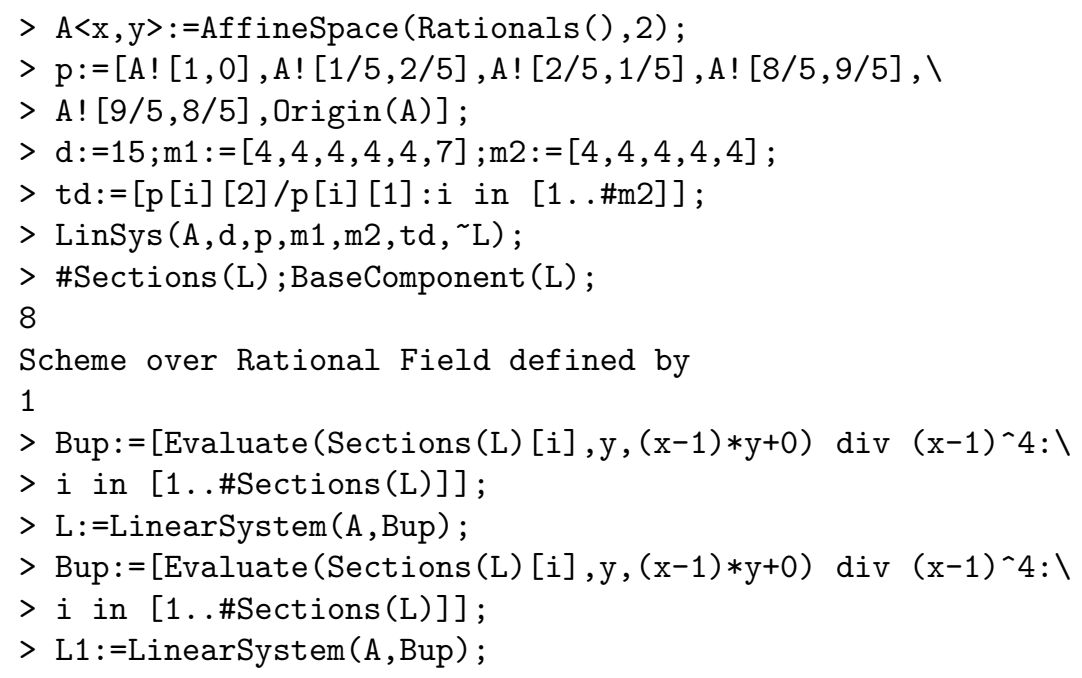

At this stage we have imposed the $(4,4)$-points and resolved $p_{1}$.

$>\mathrm{R}<\mathrm{x}, \mathrm{y}, \mathrm{u}, \mathrm{v}, \mathrm{n}\rangle:=$ PolynomialRing (Rationals ()$, 5)$;

$>\mathrm{h}:=$ hom $\langle$ PolynomialRing $(\mathrm{L} 1)->\mathrm{R}|[\mathrm{x}, \mathrm{y}]>$;

$>\mathrm{l}:=\mathrm{h}$ (Sections (L1));

Now we impose, to the elements of $l$, the necessary conditions in order to obtain the $(3,3)$-point $p_{6}=(u, v)$. The matrix $M t$ defined by these conditions cannot have maximal rank.

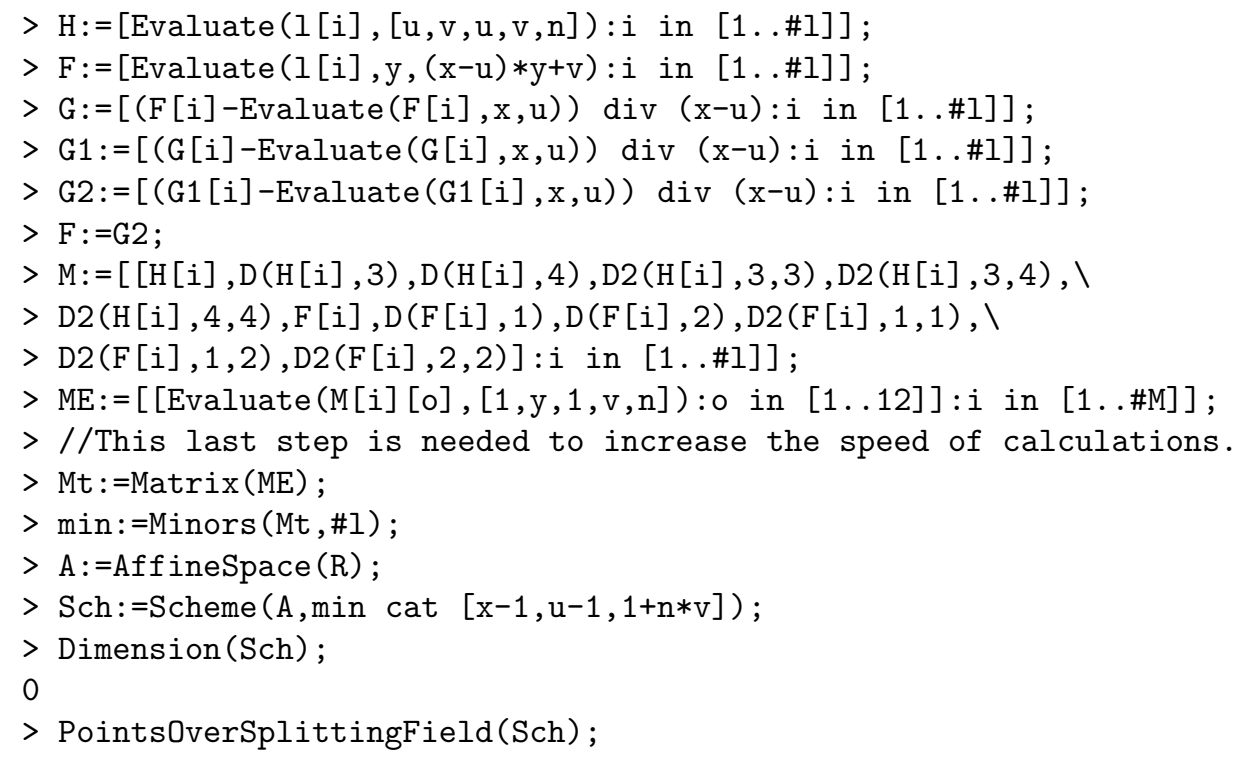


As before, this gives various solutions. We choose one which works. Here are the verifications:

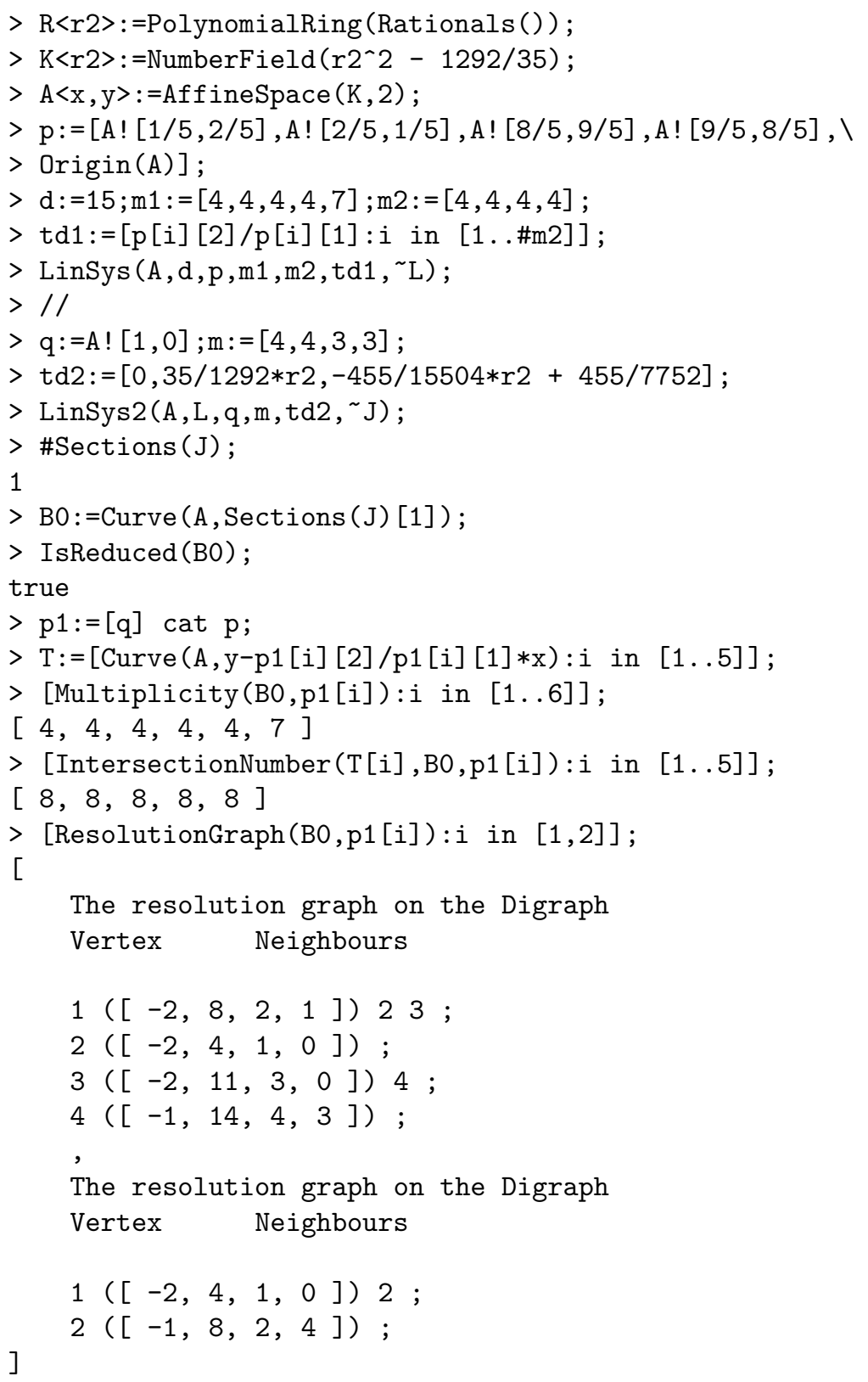

The resolution graphs for the points $p_{3}, p_{4}$ and $p_{5}$ are equal to this last one.

Now we calculate the pencil $J$ which induces the Albanese fibration.

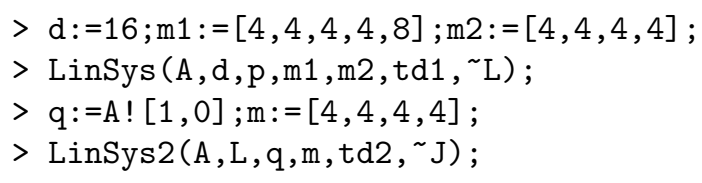


$>$ \#Sections $(\mathrm{J})$; BaseComponent $(\mathrm{J})$;

2

Scheme over $\mathrm{K}$ defined by

1

$>\mathrm{Jy}:=\mathrm{LinearSystem}(\mathrm{J}, \operatorname{Curve}(\mathrm{A}, \mathrm{y}))$;

$>$ BO eq Curve(A,Sections(Jy) [1] div y);

true

We show that the curve $B_{0}$ has no other singularities:

$>$ SingularPoints (B0);

$\{$ ( $(0,0),(1 / 5,2 / 5),(2 / 5,1 / 5),(1,0),(8 / 5,9 / 5),(9 / 5,8 / 5)$ @ $\}$

$>$ HasSingularPointsOverExtension(B0);

false

$>$ PBO:=ProjectiveClosure (B0);

$>$ Dimension(SingularSubscheme(PBO) meet LineAtInfinity(A));

$-1$

Finally we verify that the number of generators of the linear system described in Proposition 5 is 8 , which implies $K^{2}=7$.

$>\mathrm{d}:=14 ; \mathrm{m} 1:=[2,2,2,2,10] ; \mathrm{m} 2:=[4,4,4,4]$;

$>\operatorname{LinSys}(\mathrm{A}, \mathrm{d}, \mathrm{p}, \mathrm{m} 1, \mathrm{~m} 2, \mathrm{td} 1, \sim \mathrm{L})$;

$>\mathrm{m}:=[2,4,0,2]$;

$>\operatorname{LinSys} 2(\mathrm{~A}, \mathrm{~L}, \mathrm{q}, \mathrm{m}, \mathrm{td} 2, \sim \mathrm{J})$;

$>$ \#Sections $(\mathrm{J})$;

8

With this we have constructed a minimal double plane with $p_{g}=q=1, K^{2}=7$ and $g=5$.

A.2.3. $K^{2}=6$ and $g=3$. Here we give the detailed computations of Section 3.3 .

$>\mathrm{A}<\mathrm{x}, \mathrm{y}>:=$ AffineSpace (Rationals ()$, 2)$;

$>\mathrm{p}:=[\mathrm{A} ![4 / 5,7 / 5], \mathrm{A} ![-4 / 5,7 / 5], \mathrm{A} ![1,2], \mathrm{A} ![-1,2], \operatorname{Origin}(\mathrm{A})]$;

$>\mathrm{d}:=6 ; \mathrm{m} 1:=[2,2,2,2,2] ; \mathrm{m} 2:=[2,2,2,2]$;

$>t d:=[p[i][2] / p[i][1]: i$ in $[1 \ldots \# \mathrm{~m} 2]]$;

$>\operatorname{LinSys}\left(\mathrm{A}, \mathrm{d}, \mathrm{p}, \mathrm{m} 1, \mathrm{~m} 2, \mathrm{td},{ }^{\sim} \mathrm{L}\right)$;

$>$ \#Sections (L); BaseComponent (L);

2

Scheme over Rational Field defined by

1

$>\mathrm{R}<\mathrm{x}, \mathrm{y}, \mathrm{b}, \mathrm{n}>$ :=PolynomialRing(Rationals () , 4);

$>\mathrm{h}:=$ hom $<$ PolynomialRing $(\mathrm{L})->\mathrm{R} \mid[\mathrm{x}, \mathrm{y}]>$;

$>1:=\mathrm{h}($ Sections $(\mathrm{L}))$;

$>\mathrm{F}:=\mathrm{l}[1]+\mathrm{b} * 1[2]$;

$>\mathrm{G}:=$ Evaluate $(\mathrm{F}, \mathrm{x},-\mathrm{x})$;

$>C:=x^{\wedge} 2+(y-2) \wedge 2-1$;

$>/ /$

$>$ eqF $:=\mathrm{D}(\mathrm{C}, 1) * \mathrm{D}(\mathrm{F}, 2)-\mathrm{D}(\mathrm{C}, 2) * \mathrm{D}(\mathrm{F}, 1) ; / /$ To obtain a curve

$>/ /$ tangent to the conic $C$ at $p_{-} 5=(x, y)$.

$>$ eqG: $=$ Evaluate $($ eqF $, \mathrm{x},-\mathrm{x}) ; / /$ The same to $\mathrm{p}_{-} 6=(-\mathrm{x}, \mathrm{y})$.

$>$ dif: $=\mathrm{x} *(\mathrm{y}-2 * \mathrm{x}) *(\mathrm{y}-7 / 4 * \mathrm{x}) *(\mathrm{y}+2 * \mathrm{x}) *(\mathrm{y}+7 / 4 * \mathrm{x})$;

$>/ /$ We need dif to be non-zero. 


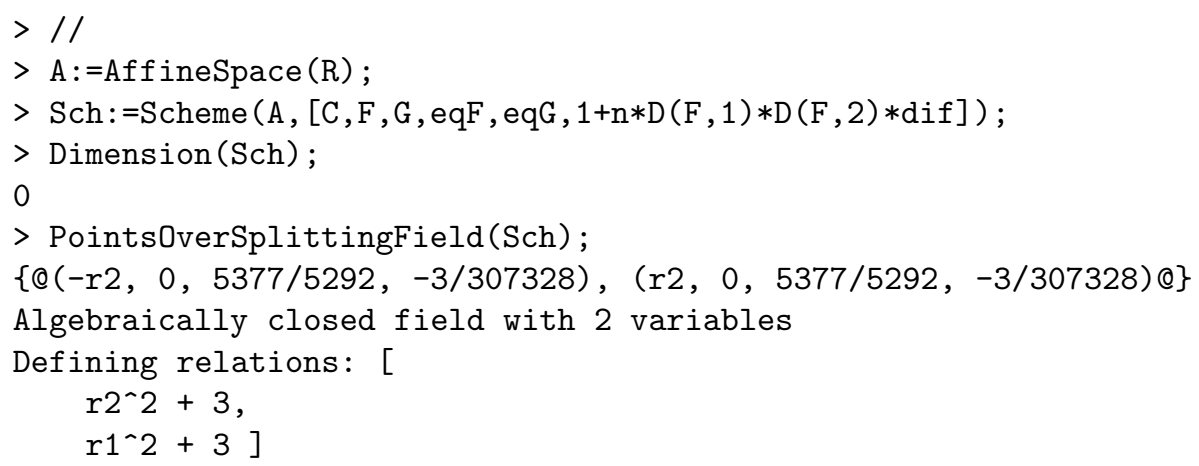

This gives the points $p_{5}, p_{6}$. We omit the remaining verifications (they are similar to the ones in A.2.1 and A.2.2.

\section{ACKNOWLEDGEMENTS}

The author wishes to thank Margarida Mendes Lopes for all the support. He is a member of the Mathematics Center of the Universidade de Trás-os-Montes e Alto Douro and is a collaborator of the Center for Mathematical Analysis, Geometry and Dynamical Systems of Instituto Superior Técnico, Universidade Técnica de Lisboa. This research was partially supported by FCT (Portugal) through Project POCTI/MAT/44068/2002.

\section{REFERENCES}

[BCP97] W. Bosma, J. Cannon, and C. Playoust, The Magma algebra system. I. The user language., J. Symbolic Comput. 24 (1997), no. 3-4, 235-265. MR.1484478

[Bea78] A. Beauville, Surfaces algébriques complexes, Astérisque, vol. 54, 1978. MR0485887 (58:5686)

[Bor07] G. Borrelli, The classification of surfaces of general type with nonbirational bicanonical map, J. Algebraic Geom. 16 (2007), no. 4, 625-669. MR.2357686 (2008m:14074)

[BPV84] W. Barth, C. Peters, and A. Van de Ven, Compact complex surfaces, vol. 4, SpringerVerlag, Berlin, 1984. MR749574 (86c:32026)

[Cat81] F. Catanese, On a class of surfaces of general type, Algebraic Surfaces, CIME, Liguori, 269-284, 1981.

[Cat99] _ Singular bidouble covers and the construction of interesting algebraic surfaces, Contemp. Math. 241, Amer. Math. Soc., 97-120, 1999. MR.1718139 (2000j:14061)

[CC91] F. Catanese and C. Ciliberto, Surfaces with $p_{g}=q=1$, Sympos. Math. 32, Academic Press, 49-79, 1991. MR.1273372 (95d:14030)

[CC93] - Symmetric products of elliptic curves and surfaces of general type with $p_{g}=$ $q=1$, J. Algebraic Geom. 2 (1993), no. 3, 389-411. MR1211993 (94i:14040)

[Cil97] C. Ciliberto, The bicanonical map for surfaces of general type, Proc. Sympos. Pure Math. 62.1, Kollár, János et al. (eds.), Algebraic geometry, 57-84, 1997. MR1492518 (98m:14040)

[CM02] C. Ciliberto and M. Mendes Lopes, On surfaces with $p_{g}=q=2$ and non-birational bicanonical map, Adv. Geom. 2 (2002), no. 3, 281-300. MR1924760 (2004d:14053)

[CP06] F. Catanese and R. Pignatelli, Fibrations of low genus. I, Ann. Scient. École. Norm. Sup. 39 (2006), no. 6, 1011-1049. MR2316980 (2008g:14014)

[Du 52] P. Du Val, On surfaces whose canonical system is hyperelliptic, Canadian J. of Math. 4 (1952), 204-221. MR.0048090 (13:977c)

[Pig] R. Pignatelli, Some (big) irreducible components of the moduli space of minimal surfaces of general type with $p_{g}=q=1$ and $k^{2}=4$, math. AG/0801.1112v1.

[Pol05] F. Polizzi, On surfaces of general type with $p_{g}=q=1, k^{2}=3$, Collect. Math. 56 (2005), no. 2, 181-234. MR2154303 (2006d:14038) 
[Pol06] Surfaces of general type with $p_{g}=q=1, k^{2}=8$ and bicanonical map of degree 2, Trans. Amer. Math. Soc. 358 (2006), no. 2, 759-798. MR2177040 (2006j:14051)

[Pol08] - On surfaces of general type with $p_{g}=q=1$ isogenous to a product of curves, Commun. Algebra 36 (2008), no. 6, 2023-2053. MR2418374 (2009c:14076)

[Pol09] , Standard isotrivial fibrations with $p_{g}=q=1$, J. Algebra 321 (2009), no. 6, 1600-1631. MR2498259

[Rei91] M. Reid, Campedelli versus Godeaux, Sympos. Math. 32, Academic Press, 309-365, 1991. MR 1273384 (95h:14031)

[Rit07] C. Rito, On surfaces with $p_{g}=q=1$ and non-ruled bicanonical involution, Ann. Scuola Norm. Sup. Pisa Cl. Sci. 6 (2007), no. 1, 81-102. MR2341516 (2008i:14058)

[Xia85] G. Xiao, Surfaces fibrées en courbes de genre deux, vol. 1137, Lecture Notes in Mathematics, Springer-Verlag, Berlin, 1985. MR872271 (88a:14042)

[Xia90] , Degree of the bicanonical map of a surface of general type, Amer. J. Math. 112 (1990), no. 5, 713-736. MR.1073006 (91i:14030)

Departamento de Matemática, Universidade de Trás-os-Montes e Alto Douro, 5000911 Vila Real, Portugal

E-mail address: crito@utad.pt 